\title{
Edge-based a Posteriori Error Estimators for Generating Quasi-optimal Simplicial Meshes
}

\author{
A. Agouzal $^{1}$, K. Lipnikov ${ }^{2}$ and Yu. Vassilevski ${ }^{3 *}$ \\ ${ }^{1}$ University Lyon1, Institute Camille Jordan, UMR 5208, 69100 Villeurbanne, France \\ ${ }^{2}$ Los Alamos National Laboratory, Los Alamos, NM 87545, U.S.A. \\ ${ }^{3}$ Institute of Numerical Mathematics, Gubkina str. 8, Moscow 119333, Russia
}

\begin{abstract}
We present a new method for generating a $d$-dimensional simplicial mesh that minimizes the $L^{p}$-norm, $p>0$, of the interpolation error or its gradient. The method uses edge-based error estimates to build a tensor metric. We describe and analyze the basic steps of our method.
\end{abstract}

Key words: finite elements, anisotropic meshes, a posteriori error estimates

AMS subject classification: 65D05, 65D15, 65N15, 65N50

\section{Introduction}

Generation of a mesh adapted to a given function requires a specially designed metric. We develop further a new methodology, proposed originally in [1, 2], for generating a tensor metric $\mathfrak{M}$ from error estimates prescribed to mesh edges. The volume and the perimeter of a $d$-simplex measured in this metric control the norm of error or its gradient. The error equidistribution principle suggests to balance $\mathfrak{M}$-volumes and $\mathfrak{M}$-perimeters to produce a $\mathfrak{M}$-quasi-uniform mesh [3, 4].

The edge-based error estimates come usually from postprocessing or a posteriori error analysis of a discrete solution. Critical advantage of edge-based error estimates over cell-based error estimates is that they provide local directional information about the error. In this paper, we consider the problem of minimizing the $P_{1}$-interpolation error or its gradient, where cell-based and edge-based errors can be easily defined. Most methods that convert the cell-based errors into a metric lose directional information. We describe a new method that uses the edge-based errors to

${ }^{*}$ Corresponding author. E-mail: yuri.vassilevski@gmail.com 
build a metric that preserves directional information. We illustrate the optimal error reduction with numerical experiments. We also summarize the existing error estimates.

\section{Edge-based interpolation error estimates}

Let $\Omega \subset \Re^{d}$ be a bounded polyhedral domain and $\Omega_{h}$ be a conformal simplicial mesh with $N_{h}$ $d$-simplexes. The volume of a $d$-simplex $\Delta$ and the total length of its edges in a metric $\mathfrak{M}$ are denoted by $|\Delta|_{\mathfrak{M}}$ and $|\partial \Delta|_{\mathfrak{M}}$, respectively. Let $\mathcal{I}_{1} u$ be the continuous piecewise linear interpolant of $u$, and $\mathcal{I}_{1, \Delta} u$ be its restriction to $\Delta$. Our goal is to generate meshes that minimize the $L^{p}$-norm, $p \in(0, \infty]$, of the interpolation error $e=u-\mathcal{I}_{1} u$ or its gradient $\nabla e$. Let us consider a $d$-simplex $\Delta$ with vertices $\mathbf{v}_{i}, i=1, \ldots, d+1$, edge vectors $\mathbf{e}_{k}=\mathbf{v}_{i}-\mathbf{v}_{j}, 1 \leq i<j \leq d+1$, and mid-edge points $\mathbf{c}_{k}, k=1, \ldots, n_{d}$, where $n_{d}=d(d+1) / 2$. Let $\lambda_{i}, i=1, \ldots, d+1$, be the linear functions on $\Delta$ such that $\lambda_{i}\left(\mathbf{v}_{j}\right)=\delta_{i j}$ where $\delta_{i j}$ is the Kronecker symbol. For every edge $\mathbf{e}_{k}$, we define the quadratic bubble function $b_{k}=\lambda_{i} \lambda_{j}$. Let $u$ be a continuous function. On each simplex $\Delta$, we consider its quadratic approximation $u_{2}=\mathcal{I}_{2, \Delta} u$, where $\mathcal{I}_{2, \Delta} u$ is the Lagrange interpolant of $u$. The interpolation error for the linear approximation of $u_{2}$ is

$$
e_{2}=u_{2}-\mathcal{I}_{1, \Delta} u_{2}=4 \sum_{k=1}^{n_{d}}\left(u_{2}\left(\mathbf{c}_{k}\right)-\mathcal{I}_{1, \Delta} u_{2}\left(\mathbf{c}_{k}\right)\right) b_{k} \equiv \sum_{k=1}^{n_{d}} \gamma_{k} b_{k}
$$

The $L^{2}$-norm of this error is given by

$$
\left\|e_{2}\right\|_{L^{2}(\Delta)}^{2}=|\Delta|(\mathbb{B} \gamma, \gamma)
$$

where $\gamma$ is the vector with $n_{d}$ components $\gamma_{k}$ and $\mathbb{B}$ is the $n_{d} \times n_{d}$ symmetric positive definite matrix with positive entries $\mathbb{B}_{k, l}=|\Delta|^{-1} \int_{\Delta} b_{k} b_{l} \mathrm{~d} V$. This error is only a number; therefore, it does not provides any directional information. To recover this information, we split this error into $n_{d}$ edge-based error estimates $\alpha_{k} \geq 0$ such that

$$
\left\|e_{2}\right\|_{L^{2}(\Delta)}=|\Delta|^{1 / 2} \sum_{k=1}^{n_{d}} \alpha_{k} \quad \text { and } \quad \sum_{k=1}^{n_{d}} \alpha_{k}=(\mathbb{B} \gamma, \gamma)^{1 / 2}
$$

In the next section, we motivate the following choice of $\alpha_{k}$ :

$$
\alpha_{k}=\left|\gamma_{k}\right|(\mathbb{B} \gamma, \gamma)^{1 / 2}\left(\sum_{k=1}^{n_{d}}\left|\gamma_{k}\right|\right)^{-1}
$$

Similarly, the $L^{2}$-norm of gradient of $e_{2}$ is given by

$$
\left\|\nabla e_{2}\right\|_{L^{2}(\Delta)}^{2}=\left\|\sum_{k=1}^{n_{d}} \gamma_{k} \nabla b_{k}\right\|_{L^{2}(\Delta)}^{2}=|\Delta|(\widetilde{\mathbb{B}} \gamma, \gamma)
$$


where $\widetilde{\mathbb{B}}$ is the symmetric positive definite matrix with entries $\widetilde{\mathbb{B}}_{k, l}=|\Delta|^{-1} \int_{\Delta} \nabla b_{k} \cdot \nabla b_{l} \mathrm{~d} V$. Again, we split the cell-based error (a number) into $n_{d}$ edge-based error estimates $\tilde{\alpha}_{k} \geq 0$ such that

$$
\left\|\nabla e_{2}\right\|_{L^{2}(\Delta)}^{2}=|\Delta| \sum_{k=1}^{n_{d}} \tilde{\alpha}_{k} \quad \text { and } \quad \sum_{k=1}^{n_{d}} \tilde{\alpha}_{k}=(\widetilde{\mathbb{B}} \gamma, \gamma)
$$

In the next section, we motivate the following choice of $\tilde{\alpha}_{k}$ :

$$
\tilde{\alpha}_{k}=\left|\gamma_{k}\right|(\widetilde{\mathbb{B}} \gamma, \gamma)\left(\sum_{k=1}^{n_{d}}\left|\gamma_{k}\right|\right)^{-1} \text {. }
$$

\section{Metric derivation from edge-based error estimates}

The next lemma shows existence of a tensor metric generated by errors associated with mesh edges.

Lemma 1. Let $\alpha_{k}, k=1, \ldots, n_{d}$, be the values prescribed to edges of a d-simplex $\Delta$ such that $\alpha_{k} \geq 0$ and $\sum_{k=1}^{n_{d}} \alpha_{k}>0$. Then, there exists a constant tensor metric $\mathfrak{M}_{\Delta}$ such that

$$
\left(\frac{d !}{(d+1)(d+2)}\right)^{1 / d}|\Delta|_{\mathfrak{M}_{\Delta}}^{2 / d} \leq \sum_{k=1}^{n_{d}} \alpha_{k} \leq|\partial \Delta|_{\mathfrak{M}_{\Delta}}^{2}
$$

The proof is sketched below and the detailed proof can be found in [2]. Let us consider the quadratic function $v_{2}=-\frac{1}{2} \sum_{k=1}^{n_{d}} \alpha_{k} b_{k}$ and denote its Hessian by $\mathbb{H}\left(v_{2}\right)$. If $\operatorname{det}\left(\mathbb{H}\left(v_{2}\right)\right) \neq 0$, we set $\mathfrak{M}_{\Delta}=\left|\mathbb{H}\left(v_{2}\right)\right|$ where $\left|\mathbb{H}\left(v_{2}\right)\right|$ is the spectral module of $\mathbb{H}\left(v_{2}\right)$. Otherwise, we increase slightly the largest $\alpha_{k}$ so that the modified function $v_{2}$ has a non-singular Hessian. In practice, increase by $1 \%$ was sufficient for all numerical experiments.

The derivation of metric $\mathfrak{M}_{\Delta}$ suggests a simple motivation for the choices (2.2) and (2.4) when $\mathbb{H}\left(v_{2}\right)$ is definite. Since the bubble function $b_{k}$ is non-zero only on one edge, we get

$$
\left(\mathfrak{M}_{\Delta} \mathbf{e}_{k}, \mathbf{e}_{k}\right)=\frac{1}{2} \alpha_{k}\left(\left|\mathbb{H}\left(b_{k}\right)\right| \mathbf{e}_{k}, \mathbf{e}_{k}\right)=4 \frac{\alpha_{k}}{\left|\gamma_{k}\right|}\left\|e_{2}\right\|_{L^{\infty}\left(\mathbf{e}_{k}\right)}
$$

When $\Delta$ is the $\mathfrak{M}_{\Delta}$-equilateral simplex, we have

$$
\frac{\alpha_{1}}{\left|\gamma_{1}\right|}\left\|e_{2}\right\|_{L^{\infty}\left(\mathbf{e}_{1}\right)}=\cdots=\frac{\alpha_{n_{d}}}{\left|\gamma_{n_{d}}\right|}\left\|e_{2}\right\|_{L^{\infty}\left(\mathbf{e}_{n_{d}}\right)}
$$

Thus, the choice (2.2) means that in a mesh consisting on $\mathfrak{M}_{\Delta}$-equilateral simplexes, we equidistribute $L^{2}$-norm of error over cells and $L^{\infty}$-norm of error over edges.

Let $\widetilde{\mathfrak{M}}_{\Delta}$ be the metric corresponding to $\tilde{\alpha}_{k}$. Repeating the above arguments, we obtain (2.4).

Combining (2.1), (2.3) and (3.1), we get the geometric representation of $L^{2}$-norm of error and its gradient:

$$
\begin{gathered}
c_{d}|\Delta|^{1 / 2}|\Delta|_{\mathfrak{M}_{\Delta}}^{2 / d} \leq\left\|e_{2}\right\|_{L^{2}(\Delta)} \leq|\Delta|^{1 / 2}|\partial \Delta|_{\mathfrak{M}_{\Delta}}^{2}, \\
\left.c_{d}|\Delta|^{1 / 2}|\Delta|\right|_{\widetilde{M}_{\Delta}} ^{1 / d} \leq\left\|\nabla e_{2}\right\|_{L^{2}(\Delta)} \leq|\Delta|^{1 / 2}|\partial \Delta|_{\widetilde{\mathfrak{M}}_{\Delta}} .
\end{gathered}
$$

In other words, both norms of the error are controlled from above by $\mathfrak{M}_{\Delta}$-perimeter and from below by $\mathfrak{M}_{\Delta}$-volume of simplex $\Delta$. Now we show how to modify the metric so that the controlling quantities will be measured in the same metric. 


\section{Generalizations to $L^{p}$-norms and $C^{2}$-functions}

Let $p \in(0 ; \infty]$. To control various $L^{p}$-norms of $e_{2}$ and $\nabla e_{2}$, we use the scaling result from [2]:

$$
\mathfrak{M}_{\Delta, p}=\left(\operatorname{det}\left(\mathfrak{M}_{\Delta}\right)\right)^{-1 /(d+2 p)} \mathfrak{M}_{\Delta} \quad \text { and } \quad \widetilde{\mathfrak{M}}_{\Delta, p}=\left(\operatorname{det}\left(\widetilde{\mathfrak{M}}_{\Delta}\right)\right)^{-1 /(d+p)} \widetilde{\mathfrak{M}}_{\Delta}
$$

Lemma 2. It holds

$$
\begin{aligned}
c|\Delta|_{\mathfrak{M}_{\Delta, p}}^{2 / d+1 / p} \leq\left\|e_{2}\right\|_{L^{p}(\Delta)} \leq C|\Delta|_{\mathfrak{M}_{\Delta, p}}^{1 / p}|\partial \Delta|_{\mathfrak{M}_{\Delta, p}}^{2}, \\
\left.\tilde{c}|\Delta|\right|_{\widetilde{\mathfrak{M}}_{\Delta, p}} ^{1 / d+1 / p} \leq \|\left.\nabla e_{2}\right|_{L^{p}(\Delta)} \leq\left.\tilde{C}|\Delta|\right|_{\widetilde{\mathfrak{M}}_{\Delta, p}} ^{1 / p}|\partial \Delta|_{\widetilde{\mathfrak{M}}_{\Delta, p}},
\end{aligned}
$$

where constants $c, C, \tilde{c}$, and $\tilde{C}$ depend only on $d$.

Up to this moment, we derived the geometric representation of various norms of $e_{2}=\mathcal{I}_{2, \Delta} u-$ $\mathcal{I}_{1, \Delta} u$. It was shown in [2] that the norm of $e_{2}$ provides a good approximation for the corresponding norm of the true error $e=u-\mathcal{I}_{1, \Delta} u$. For completeness, we summarize these important results. Let $\mathcal{F}$ be the space of symmetric $d \times d$ matrices. For a vector $\mathbf{e}_{k}$, we define the following norm:

$$
\left\|\left|\mathbf{e}_{k}\right|\right\|_{|\mathbb{H}|}^{2}=\max _{\mathbf{x} \in \Delta}\left(|\mathbb{H}(\mathbf{x})| \mathbf{e}_{k}, \mathbf{e}_{k}\right) .
$$

Then, $\|\left.|\partial \Delta|\right|_{|\mathbb{H}|} ^{2}$ means formally the sum of (4.3) over edges $\mathbf{e}_{k}$ of $\Delta$.

Lemma 3. Let $u \in C^{2}(\bar{\Delta})$. Then, there exist positive constant $c_{o}$ depending only on $d$ such that

$$
\begin{gathered}
\frac{d+1}{2 d}\left\|e_{2}\right\|_{L^{\infty}(\Delta)} \leq\left\|e_{\Delta}\right\|_{L^{\infty}(\Delta)} \leq\left\|e_{2}\right\|_{L^{\infty}(\Delta)}+\frac{1}{4} \inf _{\mathbb{F} \in \mathcal{F}}\|\partial \Delta\|_{|\mathbb{H}-\mathbb{F}|}^{2}, \\
\left\|\nabla e_{2}\right\|_{L^{\infty}(\Delta)}-c_{o} \operatorname{osc}(\mathbb{H}, \Delta) \leq\left\|\nabla e_{\Delta}\right\|_{L^{\infty}(\Delta)} \leq\left\|\nabla e_{2}\right\|_{L^{\infty}(\Delta)}+c_{o} \operatorname{osc}(\mathbb{H}, \Delta),
\end{gathered}
$$

where

$$
\operatorname{osc}(\mathbb{H}, \Delta)=\frac{|\partial \Delta|^{d-1}}{|\Delta|} \inf _{\mathbb{F} \in \mathcal{F}}|| \partial \Delta \mid \|_{|\mathbb{H}-\mathbb{F}|}^{2} \cdot
$$

The oscillation terms are conventional in contemporary error analysis. Their value depend on the simplex and particular features of the function. For instance, if $u \in C^{2}(\bar{\Delta})$, and $\Delta$ is shape regular, one has osc $(\mathbb{H}, \Delta) \leq C|\partial \Delta| \inf _{\mathbb{F} \in \mathcal{F}}|\mathbb{H}-\mathbb{F}|_{\infty}$. Thus, the oscillation terms are smaller than the errors. Similar analysis can be performed for $L^{p}$-norms, $p>0$.

\section{Asymptotic error estimates}

Let $\Omega_{h}$ and $\tilde{\Omega}_{h}$ be simplicial meshes with $N_{h}$ cells that balance the volume and perimeter of cells:

$$
\begin{aligned}
N_{h}^{-1}|\Omega|_{\mathfrak{M}_{p}} \simeq|\Delta|_{\mathfrak{M}_{\Delta, p}} \simeq|\partial \Delta|_{\mathfrak{M}_{\Delta, p}}^{d} \quad \forall \Delta \in \Omega_{h}, \\
N_{h}^{-1}|\Omega|_{\widetilde{\mathfrak{M}}_{p}} \simeq|\Delta|_{\widetilde{\mathfrak{M}}_{\Delta, p}} \simeq|\partial \Delta|_{\mathfrak{M}_{\Delta, p}}^{d} \quad \forall \Delta \in \tilde{\Omega}_{h} .
\end{aligned}
$$


On such meshes, the following error estimates are held

$$
\begin{aligned}
\|e\|_{L^{p}(\Omega)} & =\left(\sum_{\Delta \in \Omega_{h}}\|e\|_{L^{p}(\Delta)}^{p}\right)^{\frac{1}{p}} \lesssim\left(\sum_{\Delta \in \Omega_{h}}|\Delta|_{\mathfrak{M}_{\Delta, p}}^{1+\frac{2 p}{d}}\right)^{\frac{1}{p}} \lesssim|\Omega|_{\mathfrak{M}_{p}}^{\frac{1}{p}+\frac{2}{d}} N_{h}^{-\frac{2}{d}}, \\
\|\nabla e\|_{L^{p}(\Omega)} & =\left(\sum_{\Delta \in \tilde{\Omega}_{h}}\|\nabla e\|_{L^{p}(\Delta)}^{p}\right)^{\frac{1}{p}} \lesssim\left(\sum_{\Delta \in \tilde{\Omega}_{h}}|\Delta|_{\widetilde{\mathfrak{M}}_{\Delta, p}}^{1+\frac{p}{d}}\right)^{\frac{1}{p}} \lesssim|\Omega|_{\mathfrak{M}_{p}}^{\frac{1}{p}+\frac{1}{d}} N_{h}^{-\frac{1}{d}},
\end{aligned}
$$

where $a \lesssim b$ means existence of constants $c$ and $C$ independent of mesh such that $c a \leq b \leq C a$. In other words, the $\mathfrak{M}_{p}$ (resp., $\widetilde{\mathfrak{M}}_{p}$ )-quasi-uniform meshes provide asymptotically optimal rate for reduction of the $L^{p}$-norm of the error (resp., the gradient of the error).

\section{Metric-based mesh adaptation}

We use Algorithm 1 to build an adaptive mesh minimizing the $L^{p}$-norm of error or its gradient. It provides faster convergence and results in smoother meshes when the metric is continuous. To define a continuous metric we suggest a method of shifts. For every node $\mathbf{a}_{i}$ in $\Omega_{h}$, we define the superelement $\sigma_{i}$ as the union of all $d$-simplexes sharing $\mathbf{a}_{i}$. Then, $\mathfrak{M}\left(\mathbf{a}_{i}\right)$ is defined as one of the metrics in $\sigma_{i}$ with the largest determinant. This method always chooses the worst metric in the superelement. To generate a $\mathfrak{M}$-quasi-uniform mesh, we use local mesh modifications described in [3] and implemented in package Ani2D (sourceforge.net/projects/ani2d).

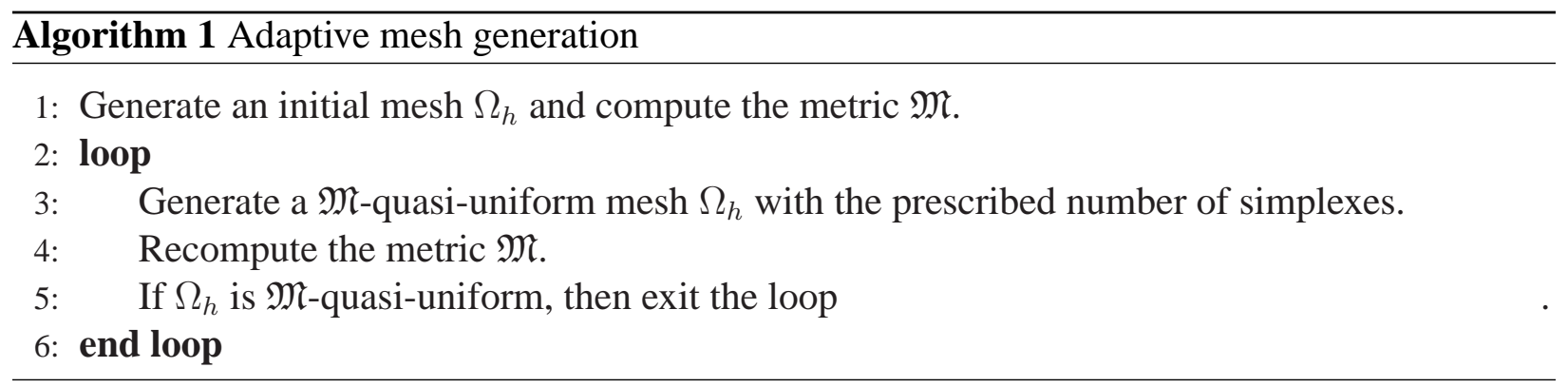

For the numerical illustration, we consider the analytical function

$$
u=\left(x^{2} y+y^{3}\right) / 16^{3}+\tanh (2(\sin (6 y)-3 x)(\sin (6 x)-3 y))
$$

in the Texas-shape domain inscribed in $\left[-\frac{3}{2} ; \frac{3}{2}\right]$. The spider-like isolines of $u$ (see Fig. 1) show that this function has both isotropic and anisotropic regions. Table 1 verifies the theoretical estimates from Section 5. The $L^{\infty}$-norm of the interpolation error on meshes $\Omega_{h}$ built with Algorithm 1 and metric $\mathfrak{M}_{p}$ is proportional to $N_{h}^{-1}$. The $L^{\infty}$-norm of its gradient on meshes $\tilde{\Omega}_{h}$ built with Algorithm 1 and metric $\widetilde{\mathfrak{M}}_{p}$ is proportional to $N_{h}^{-1 / 2}$. The last column in Table 1 shows that the $L^{\infty}$-norm of the interpolation error on mesh $\tilde{\Omega}_{h}$, which is not the optimal mesh for this error, exhibits still the optimal convergence rate; albeit, the error is larger than that on mesh $\Omega_{h}$. We also note that the mesh $\tilde{\Omega}_{h}$ (Fig.1, right) is denser than the mesh $\Omega_{h}$ (Fig.1, left) in regions where the solution is sharp. 


\begin{tabular}{c|c|c|c}
$N_{h}$ & $\|e\|_{L^{\infty}\left(\Omega_{h}\right)}$ & $\|\nabla e\|_{L^{\infty}\left(\tilde{\Omega}_{h}\right)}$ & $\|e\|_{L^{\infty}\left(\tilde{\Omega}_{h}\right)}$ \\
\hline 2000 & $3.72 \mathrm{e}-2$ & $1.23 \mathrm{e}+0$ & $2.04 \mathrm{e}-1$ \\
4000 & $1.76 \mathrm{e}-2$ & $8.00 \mathrm{e}-1$ & $9.24 \mathrm{e}-2$ \\
8000 & $8.12 \mathrm{e}-3$ & $5.36 \mathrm{e}-1$ & $4.06 \mathrm{e}-2$ \\
16000 & $4.60 \mathrm{e}-3$ & $4.00 \mathrm{e}-1$ & $1.99 \mathrm{e}-2$ \\
32000 & $2.19 \mathrm{e}-3$ & $2.72 \mathrm{e}-1$ & $1.11 \mathrm{e}-2$ \\
64000 & $1.22 \mathrm{e}-3$ & $1.92 \mathrm{e}-1$ & $6.47 \mathrm{e}-3$ \\
\hline rate & 0.99 & 0.53 & 1.00
\end{tabular}

Table 1: Convergence of the $L^{\infty}$-norm of the interpolation error and its gradient.
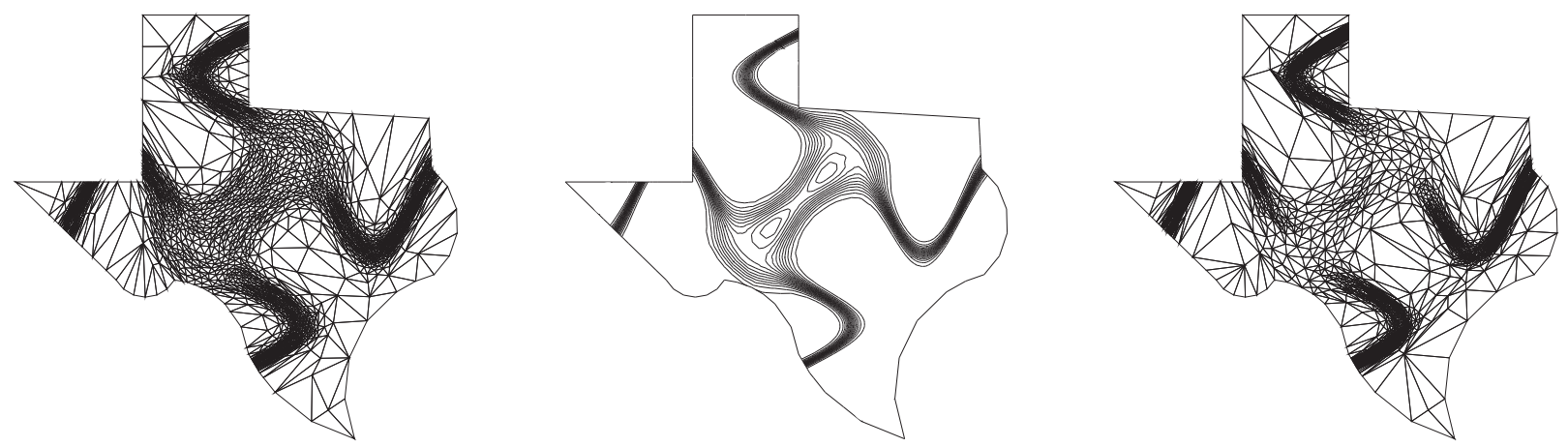

Figure 1: Left: The mesh $\Omega_{h}$ with roughly 2000 triangles minimizing $\|e\|_{L^{\infty}\left(\Omega_{h}\right)}$. Middle: The isolines of function (6.1). Right: The mesh $\tilde{\Omega}_{h}$ with roughly 2000 triangles minimizing $\|\nabla e\|_{L^{\infty}\left(\tilde{\Omega}_{h}\right)}$.

\section{Acknowledgements}

This research was partly supported by the Russian Foundation for Basic Research through grant 08-01-00159-a and by RAS program "Optimal methods for problems of mathematical physics".

\section{References}

[1] A. Agouzal, K. Lipnikov, Y. Vassilevski. Generation of quasi-optimal meshes based on a posteriori error estimates. In: Proceedings of 16th International Meshing Roundtable. M.Brewer and D.Marcum (eds.), Springer, (2007), 139-148.

[2] A. Agouzal, K. Lipnikov, Y. Vassilevski. Hessian-free metric-based mesh adaptation via geometry of interpolation error. To appear in Comp. Math. Math. Phys., 50 (2010).

[3] Y. Vassilevski, K. Lipnikov. Adaptive algorithm for generation of quasi-optimal meshes. Comp. Math. Math. Phys., 39 (1999), 1532-1551.

[4] Y. Vassilevski, A. Agouzal. An unified asymptotic analysis of interpolation errors for optimal meshes. Doklady Mathematics, 72 (2005), 879-882. 